\title{
An Asymptotic Study of the Steady State Model of Oxygen Diffusion in Tissue Regions
}

\author{
Kartika Yulianti ${ }^{1} \&$ Agus Y. Gunawan ${ }^{2}$ \\ ${ }^{1}$ Department of Mathematics Education, Indonesia University of Education (UPI), \\ Jalan Dr. Setiabudhi No. 229 Bandung, Jawa Barat 40154, Indonesia \\ ${ }^{2}$ Industrial and Financial Mathematics Research Group, Faculty of Mathematics and \\ Natural Sciences, Institut Teknologi Bandung, Jalan Ganesha 10 Bandung, \\ Jawa Barat 40132, Indonesia \\ Email: ykar_tika@yahoo.com, aygunawan@math.itb.ac.id
}

\begin{abstract}
Oxygen plays an important role in the metabolism of cells inside the human body. The transfer of oxygen from blood to tissue takes place in capillaries through a diffusion process. The capillary-tissue region is usually represented by the so-called Krogh cylinder model, in which the distribution of the oxygen concentration in a tissue region leads to a diffusion equation with oxygen consumption rates following the Michaelis-Menten kinetics. In this paper, we restrict ourselves to the steady state case and solve the equation analytically by means of asymptotic expansion for a particular limit of the oxygen consumption rate. Results show that there exists a critical ratio between supply and consumption of oxygen in the tissue region in order to fulfill the cell's oxygen requirements. Above from this critical ratio, we also found a critical distance in the tissue region above which the oxygen concentration vanishes. We compared our asymptotic results with numerical simulations, which turned out to be quite in agreement.
\end{abstract}

Keywords: asymptotic expansions; diffusion process; Krogh cylinder.

\section{$1 \quad$ Introduction}

In the circulatory system, arteries and veins are responsible for the transport of blood to and from the heart. An artery carries oxygen-rich blood until it encounters a capillary bed. In the capillaries, the oxygen diffuses into the surrounding tissue where it is absorbed. The deoxygenated blood goes back to the heart through a vein (see in detail in Middleman [1]). If the supply of oxygen to the tissue is insufficient then bodily functions will be impaired and damage can result; for example, if the brain is involved, fainting may occur.

The first attempt to describe the phenomenon of oxygen supply to living tissue through microcirculation of blood in mathematical terms is the classical Krogh model [2]. This model deals with an idealized geometrical arrangement, consisting of a circular cross section of one capillary concentric with a circular

Received October $6^{\text {th }}, 2011$, Revised February $1^{\text {st }}, 2012$, Accepted for publication June $22^{\text {nd }}, 2012$.

Copyright (C) 2012 Published by LPPM ITB, ISSN: 1978-3043, DOI: 10.5614/itbj.sci.2012.44.2.6 
cross section of muscle tissue. The derivation of the model can also be found in Middleman [1], in the form of a diffusion equation. To some extent, the role of oxygen transport to tissue has attracted much attention from some authors. Among them, Salathe and Xu [3] studied the nonlinear model describing the role hemoglobin and myoglobin play in facilitating oxygen transport to tissue, using multiple scale techniques and similarity transformation. Titkombe and Ward [4] investigated an asymptotic solution for the diffusion problem in two dimensions, which describes oxygen transport from multiple capillaries to skeletal muscle tissue. They demonstrated that for $\mathrm{N}$ capillaries with a small but arbitrary cross-sectional shape, the problem leads to a singular perturbation problem that involves an infinite expansion of logarithmic terms of the small parameter, which characterizes the capillary cross sections. Salathe investigated the analysis of the oxygen concentration in a two-dimensional array of capillaries in the form of a system of ordinary differential equations for the oxygen concentration, coupled with a system of algebraic equations for the fluxes [5]. She calculated the oxygen concentration within an array of capillaries for the case when each capillary has a different initial concentration and for the case when each capillary has a different flow rate. The existence of a global, unique and smooth solution for the nonlinear diffusion of oxygen in living tissue in presence of consumption due to metabolism, was discussed by Mikelic and Primicerio [6].

The aim of the present study is to investigate the solution of the steady state case by the perturbation technique, i.e. an asymptotic expansion, which is not discussed by Middleman [1]. Here, a parameter arising from the MichaelisMenten kinetics that represents the Michaelis constant is considered to be small compared to the oxygen concentration in the tissue region. We also predict the bound of the ratio of the oxygen consumption rate to the oxygen supply, in which this parameter measures the availability of oxygen concentration in the tissue.

This paper is organized as follows: in Section 2 we present an oxygen diffusion model taken from Middleman [1]; this section is devoted in order to be selfcontained during our discussion. In Section 3 the steady state case is discussed; the equation is solved analytically by asymptotic expansion. Here, the interior boundary layer problem is proposed and solved by the match asymptotic expansion technique. Also, a numerical simulation is worked out to confirm our asymptotic solution. Conclusions are written in the last section.

\section{The Model}

The most common geometrical representation of the capillary-tissue region is the Krogh cylinder arrangement as shown in Figure 1. This model introduces 
the concept of a repetitive unit structure. With such a unit structure one implies that each section of capillaries is responsible for the supply of oxygen to a corresponding cylindrical section of the surrounding tissue. This is a great oversimplification of the real situation. Each section of tissue is actually supplied to some extent by many surrounding capillaries, although the supply must surely be provided mostly by the closest capillaries.

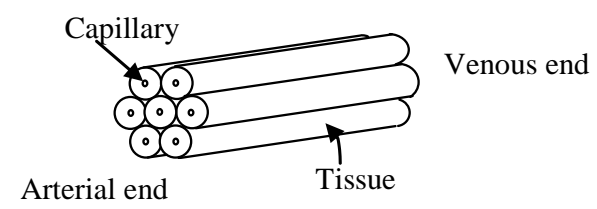

Figure 1 Krogh cylinder arrangement.

In order to yield a model amenable to solution, assumptions have to be made. Following Middleman [1], the limits of the model are summarized as follows:

1. The Krogh cylinder is assumed to be an appropriate model for the geometry. The chemical reactions taking place in the tissue surrounding the capillary are continuously distributed.

2. Symmetry about the axis of the capillary-tissue cylinder is allowed. This model suggests that there is a cylindrical surface about a capillary across which transport from surrounding capillaries just balances transport toward these capillaries. Hence, net transport rates vanish along this surface, which can be called a "no-flux" cylinder.

Basically, there are two transport regions for blood circulation: within the capillary and outside of the capillary (the tissue region). Within the capillary, the material is transported through the blood by both convection and diffusion. In the tissue region the material moves only by molecular diffusion (see Figure 2). In our paper, we will restrict ourselves to the tissue region.

Based on the assumptions above, Middleman [1] obtained a diffusion equation in the tissue region given by

$$
\frac{\partial \tilde{c}}{\partial \tilde{t}}=D\left(\frac{1}{\tilde{r}} \frac{\partial \tilde{c}}{\partial \tilde{r}}+\frac{\partial^{2} \tilde{c}}{\partial \tilde{r}^{2}}\right)-g(\tilde{c}), \quad a \leq \tilde{r} \leq b,
$$

where $\tilde{c}>0$ is the oxygen concentration, $\tilde{r}$ is radial direction, $\tilde{t}$ is mean time, $D$ is the diffusion coefficient, and $g(\tilde{c})$ is oxygen consumption rate. In (1), axial diffusion of oxygen was neglected since it is beyond our interest, and $D$ 
was assumed to be constant. It is often observed that oxygen consumption rate $g(\tilde{c})$ follows the Michaelis-Menten kinetics, which may be expressed as

$$
g(\tilde{c})=\frac{A \tilde{c} H(\tilde{c})}{B+\tilde{c}}
$$

where $A$ is the maximal reaction rate constant, and $B$, the so-called Michaelis constant, is the oxygen concentration that yields the half-maximal reaction rate [7]. In (2), $H(\tilde{c})$ denotes the Heaviside function. The presence of this function is naturally understood since the reaction only takes place when $\tilde{c}(\tilde{r})$ is still available. We note that the rate of oxygen conversion is $(A / B) \tilde{c}$ for small concentration levels compared to $B$, and saturates at a rate of $\mathrm{A}$ which is independent of $\tilde{c}$ for large concentration levels. It is usually the case that concentration levels are large compared to $\mathrm{B}$ and this is the case we will examine here.

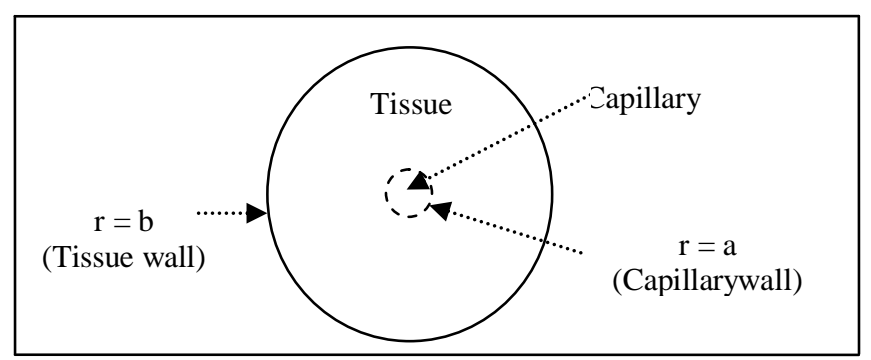

Figure 2 Capillary-Tissue Region. Our domain of interest is the tissue region.

Eq. (1) must be supplemented by boundary conditions. We propose the boundary conditions as follows. For $\tilde{t}=0$ we assume that the concentration of oxygen in all tissue regions is $c_{a}$,

$$
\tilde{c}(\tilde{r}, 0)=c_{a},
$$

For $\tilde{t}>0$, the capillary supplies a flow of blood having a constant concentration $c_{i}$, where $c_{i}>c_{a}$. This inequality allows the diffusion process to occur. Furthermore, during time $\varepsilon$, where $\varepsilon$ is a fixed time, i.e. $0 \leq \tilde{t}<\varepsilon$, we propose that the concentration of oxygen in the capillary wall increases linearly from $c_{a}$ to $c_{i}$, and we assume that for $\tilde{t} \geq \varepsilon$ the continuity of the oxygen concentration at $\tilde{r}=a$ is achieved. Therefore, the boundary conditions in the capillary wall, $\tilde{r}=a$, are chosen to be 


$$
\tilde{c}(a, \tilde{t})=\left\{\begin{array}{lr}
\frac{c_{i}-c_{a}}{\varepsilon} \tilde{t}+c_{a}, & 0 \leq \tilde{t}<\varepsilon \\
c_{i}, & \tilde{t} \geq \varepsilon
\end{array}\right.
$$

At $\tilde{r}=b$, the no-flux condition is satisfied,

$$
\frac{\partial \tilde{c}}{\partial \tilde{r}}(b)=0 .
$$

The model is now described by (1)-(5). Note that $\tilde{c}$ is the mean concentration of oxygen. Therefore, it has to be positive.

In the next section, we will solve the steady state equation analytically by means of asymptotic expansion.

\section{$3 \quad$ Steady State Case: Asymptotic Solution and Numerical Simulations}

For this condition, the concentration is in stationary equilibrium. Therefore, the model is reduced to

$$
\begin{aligned}
& D\left(\frac{1}{\tilde{r}} \frac{\partial \widetilde{c}}{\partial \tilde{r}}+\frac{\partial^{2} \tilde{c}}{\partial \tilde{r}^{2}}\right)=\frac{A \widetilde{c} H(\tilde{c})}{B+\tilde{c}}, \quad a \leq \tilde{r} \leq b \\
& \tilde{c}(a)=c_{i} \\
& \frac{\partial \tilde{c}}{\partial \tilde{r}}(b)=0
\end{aligned}
$$

For convenience, we transform the model into dimensionless form, as follows. Let us take

$$
\widetilde{c}=c_{i} c, \quad \widetilde{r}=a v
$$

Eqs. (6)-(8) now become

$$
\begin{aligned}
& \frac{1}{r} \frac{\partial}{\partial r}\left(r \frac{\partial c}{\partial r}\right)=\frac{\lambda c H(c)}{\alpha+c}, \quad 1 \leq r \leq \frac{b}{a} \\
& c(1)=1 \\
& \frac{\partial c}{\partial r}\left(\frac{b}{a}\right)=0,
\end{aligned}
$$


where $\lambda=\frac{A a^{2}}{D c_{i}}, \alpha=\frac{B}{c_{i}}$.

Note that $\lambda$ is the scaled maximal reaction rate constant and $\alpha$ is the scaled Michaelis constant. Eq. (10) supplemented by (11) and (12) will be solved, both analytically and numerically. Numerical simulations will be carried out in order to confirm our analytical results. For the analytical part, we will apply asymptotic expansions (see Holmes [8]); for the numeric part, we will use the finite difference method (see Matthews [9]).

\subsection{Asymptotic Analysis}

The solution of (10)-(12) is unique and non-negative (see Logan [10]). Here, we consider the behavior of the solution for the particular limit of $\lambda=O(1)$ and $\alpha \rightarrow 0$. Physically, $\lambda=O(1)$ may represent the condition where the magnitude of the reaction rate is of the same order as the diffusion rate, and $\alpha \rightarrow 0$ may represent the condition for which the Michaelis constant is very small compared to the oxygen concentration in the tissue region. This is an interesting limit because, as we will see, there is a critical value of $\lambda$, above which a deoxygenation region exists. The line to solve the present case is similar to that of Mattheij, et al. [11], but applied to a different case and different domain of interest. Mattheij, et al. [11] studied a catalytic reaction problem in pellets. The equation was written in spherical coordinates for $0<r<R$ with no-flux condition at $r=0$. Here, the equation is derived to describe the diffusion process in tissue regions and is presented in cylindrical coordinates, where the domain of the problem is $1 \leq r \leq \frac{b}{a}$ with no-flux condition at $r=\frac{b}{a}$.

From boundary condition (11), we observe $c(1)=O(1)$, at least in the neighborhood of $r=1$. Then, we may estimate $c=O(1)$. So, we expand $c$ as

$$
c(r ; \alpha)=c_{0}(r)+\alpha c_{1}(r)+O\left(\alpha^{2}\right) .
$$

Substitution of (13) in (10) yields

$$
\frac{1}{r}\left(r c_{0}^{\prime}\right)^{\prime}+\alpha \frac{1}{r}\left(r c_{1}^{\prime}\right)^{\prime}+O\left(\alpha^{2}\right)=\frac{\lambda c}{\alpha+c}=\lambda-\frac{\alpha \lambda}{c_{0}}+O\left(\alpha^{2}\right)
$$

In (14), the prime denotes the derivative with respect to $r$. Note that when $c(r ; \alpha)$ is getting closer to zero, i.e. $c \leq O(\alpha)$, the neglected term (to the first order) on the last expression in (14) is larger than the retained term, i.e. for a 
small $c_{0}$ the term $\alpha \lambda / c_{0}$ will not be small and will contribute to the first term. Thus, expansion (13) is not valid anymore. For that situation we have to do more, which will be explained later on.

To leading order, from (14) we get

$$
\frac{1}{r}\left(r c_{0}^{\prime}\right)^{\prime}=\lambda
$$

and obtain

$$
c_{0}(r)=\frac{\lambda}{4} r^{2}+K_{1} \ln r+K_{2}
$$

where $K_{1}$ and $K_{2}$ are constants to be determined. Applying $c_{0}(1)=1$, we find

$$
c_{0}(r)=\frac{\lambda}{4} r^{2}+K_{1} \ln r+1-\frac{\lambda}{4} .
$$

If $c_{0}$ is a valid approximation everywhere, we can directly apply the boundary condition $c_{0}^{\prime}(b / a)=0$ and obtain $K_{1}=-\frac{\lambda b^{2}}{2 a^{2}}$. Thus, (17) becomes

$$
c_{0}(r)=1-\lambda\left(\frac{b^{2}}{2 a^{2}} \ln r-\frac{r^{2}-1}{4}\right) .
$$

From (18), $c_{0}$ will be positive if

$$
\lambda<\frac{1}{\frac{b^{2}}{2 a^{2}} \ln r-\frac{r^{2}-1}{4}}=F(r)
$$

The graph of $F(r)$ is given in Figure 3, for $\frac{b}{a}=11$. The dashed line represents a critical value $\lambda_{c r}=F(b / a)$ below which $c_{0}$ will be positive. From this figure we note that above $\lambda_{c r}$ there will be a point where $c_{0}$ is zero; this case will be considered in the following. 


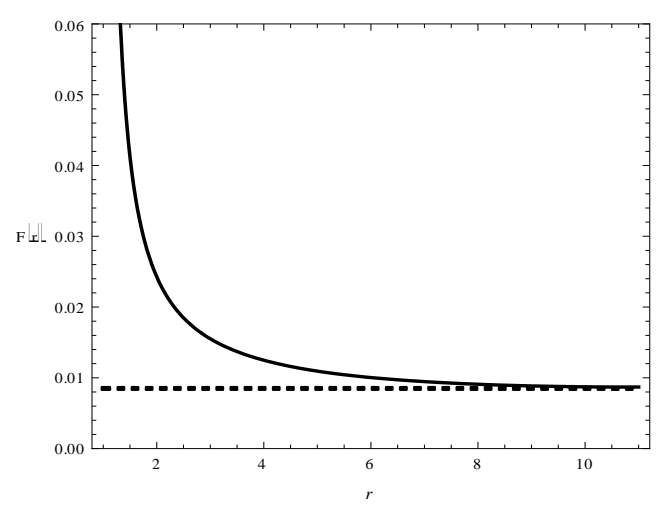

Figure 3 Graph of $F(r)$. The dashed line represents critical value $\lambda_{c r}=F(b / a)$.

Now, let us consider when $c(r ; \alpha)$ is getting closer to zero, $0 \leq c \leq O(\alpha)$. Suppose $c_{0}$ is zero at $r_{1}$. Here, $c_{0} \geq 0$ for $1 \leq r \leq r_{1}$ and $c_{0}<0$ for $r_{1}<r \leq \frac{b}{a}$. Above $r_{1}$, the (negative) solution is certainly not valid. We scale $c$ as $c=\alpha^{n} \bar{c}, n \geq 1$. Then, Eq. (10) becomes:

$$
\alpha \frac{1}{r} \frac{d}{d r}\left(r \frac{d \bar{c}}{d r}\right)=\frac{\lambda \bar{c}}{1+\alpha^{n-1} \bar{c}} .
$$

Since $\alpha \rightarrow 0$ and $n \geq 1$, each term in the following asymptotic expansion

$$
\bar{c}(r ; \alpha)=\bar{c}_{0}(r)+\ldots, r \geq r_{1}
$$

has to be zero. So, the solution for $r_{1} \leq r \leq \frac{b}{a}$ is asymptotically equivalent to zero. What remains to be determined are the position and neighborhood of the transition point $r_{1}$. As noted before, this point is still unknown since we do not know the constant $K_{1}$ yet. Now we shall deal with the interior layer problem, that will be worked out by the matched asymptotic expansion technique. Let us write the following expression

$$
c=\alpha^{n} \psi, \quad r=r_{1}+\alpha^{m} \xi .
$$

Substituting (22) into (10), we find 


$$
\frac{d^{2} \psi}{d \xi^{2}}+\frac{\alpha^{m}}{r_{1}+\alpha^{m} \xi} \frac{d \psi}{d \xi}=\frac{\lambda \alpha^{2 m} \psi}{\alpha+\alpha^{n} \psi}=\frac{\lambda \alpha^{2 m-1} \psi}{1+\alpha^{n-1} \psi}
$$

Since $m, n>0$, from the distinguished limit we obtain $n=1$ and $m=\frac{1}{2}$. Thus,

$$
c=\alpha \psi, \quad r=r_{1}+\sqrt{\alpha} \xi,
$$

and to order $\sqrt{\alpha}$

$$
\frac{d^{2} \psi}{d \xi^{2}}=\frac{\lambda \psi}{1+\psi}
$$

Now, we have to apply the matching condition. At the right side of $r_{1}, \psi$ has to connect to $c(r)=0$, so $\psi \rightarrow 0$, if $\xi \rightarrow \infty$. At the left side of $r_{1}, \psi$ has to connect to $c_{0}(r)$, the behavior of which near $r_{1}(\xi \rightarrow-\infty)$ is described by:

$$
\begin{aligned}
c_{0}(r) & =c_{0}\left(r_{1}\right)+\left(r-r_{1}\right) c_{0}^{\prime}\left(r_{1}\right)+\frac{1}{2}\left(r-r_{1}\right)^{2} c_{0}^{\prime \prime}\left(r_{1}\right)+\ldots \\
& =\sqrt{\alpha} \xi c_{0}^{\prime}\left(r_{1}\right)+\frac{1}{2} \alpha \xi^{2} c_{0}^{\prime \prime}\left(r_{1}\right)+\ldots \\
& \cong \alpha \psi(\xi) .
\end{aligned}
$$

The $O(\sqrt{\alpha})$-term has to be equal to zero. So, $c_{0}^{\prime}\left(r_{1}\right)=0$. Physically, this result makes sense: if there is no source at the border of the region where the concentration vanishes, then the flux there cannot be anything else but zero. Hence, we have

$$
0=c_{0}^{\prime}\left(r_{1}\right)=\frac{\lambda r_{1}}{2}+\frac{K_{1}}{r_{1}}
$$

which implies $K_{1}=-\frac{\lambda r_{1}^{2}}{2}$. We finally obtain

$$
c_{0}(r)=1-\lambda\left(\frac{r_{1}^{2}}{2} \ln r-\frac{r^{2}-1}{4}\right),
$$

where $r_{1}$ is the root of $c_{0}(r)$. 


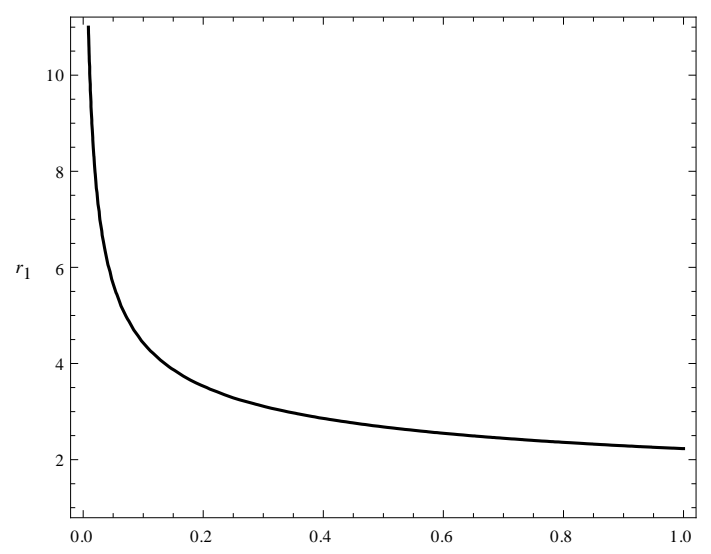

Figure $4 r_{1}$ as a function of $\lambda$.

Figure 4 shows the graph of $r_{1}$ as a function of $\lambda$.

Remarks. Eq. (25) can be rewritten as $U \frac{d U}{d \psi}=\frac{\lambda \psi}{1+\psi}$ where $U=\frac{d \psi}{d \xi}$ and yields $\frac{U^{2}}{2}=\lambda(\psi-\ln |1+\psi|+C)$ with $C=0$ giving the required zero condition on $\psi$. Note that for this required (inner) solution we also have $U=0$. For matching between the inner and outer solutions, we have $U \rightarrow \sqrt{2 \lambda \psi}$ which says that the outer solution is required to vanish to order $\sqrt{\alpha}$ as $r \rightarrow r_{1}$. This shows that to the first order the results are consistent.

To recapitulate, for $\lambda<\lambda_{c}$ the solution $c_{0}(r)$ is

$$
c_{0}(r)=1-\lambda\left(\frac{b^{2}}{2 a^{2}} \ln r-\frac{r^{2}-1}{4}\right),
$$

and for $\lambda \geq \lambda_{c}$ the solution is

$$
c_{0}(r)= \begin{cases}1-\lambda\left(\frac{r_{1}^{2}}{2} \ln r-\frac{r^{2}-1}{4}\right), & \text { if } 1 \leq r<r_{1} ; \\ 0, & \text { if } r_{1} \leq r \leq \frac{b}{a} .\end{cases}
$$

Next, we will compare our asymptotic solutions with numerical simulations. 


\subsection{Numerical Solution}

We will apply the finite difference method. Interval $\left[1, \frac{b}{a}\right]$ is divided into $n$ grid, with size $h=\frac{(b / a-1)}{n}$. So we have grid points $1=r_{0}<r_{1}<\ldots<r_{n}=\frac{b}{a}$, with $r_{j}=1+j h$. For simplicity, concentration $c\left(r_{j}\right)$ is denoted by $c_{j}$. For approximation of the $\frac{d c}{d r}$ and $\frac{d^{2} c}{d r^{2}}$ at $r_{j}$, we use the Taylor expansion. Therefore, the schemes for (10)-(12) are:

$$
\begin{aligned}
& v_{j} c_{j+1}-\frac{2}{h^{2}} c_{j}+\varsigma_{j} c_{j-1}=\frac{\lambda c_{j} H\left(c_{j}\right)}{\alpha+c_{j}}, \quad j=1, \ldots n,-1 \\
& c_{j}=1 \quad \text { for } j=0, \\
& \frac{2\left(c_{j-1}-c_{j}\right)}{h^{2}}=\frac{\lambda c_{j} H\left(c_{j}\right)}{\alpha+c_{j}} \quad \text { fo } \mathrm{r} j=n .
\end{aligned}
$$

where $v_{j}=\frac{1}{h^{2}}+\frac{1}{2 h r_{j}}$ and $\varsigma_{j}=\frac{1}{h^{2}}-\frac{1}{2 h r_{j}}$.

Finally, we use Newton Raphson Method to solve the nonlinear system (27)(29). Figures 5 and 6 show the curves of the asymptotic (solid line) and numerical solutions (dotted line) for $b / a=11$.This size ratio is taken from the data given by Middleman [1]. For this size ratio we find $\lambda_{c r}=0.009$

In Figure 5, the solutions are plotted for $\lambda=1$ and for various values of $\alpha$. We observe that the smaller the value of $\alpha$, the better the approximation; for $\alpha=0.001$ the asymptotic and the numerical solutions hardly differ from that of $\alpha=0.5$. Since $\lambda=1>\lambda_{c r}$ there exists a deoxygenation region. Here, we find $r_{1}=2.23$.

Figure 6 shows the curves of the solutions, for $\alpha=0.001$ and for various values of $\lambda$. Both solutions are perfectly in agreement. We also note that the smaller the value of $\lambda$, the narrower the deoxygenation region. 

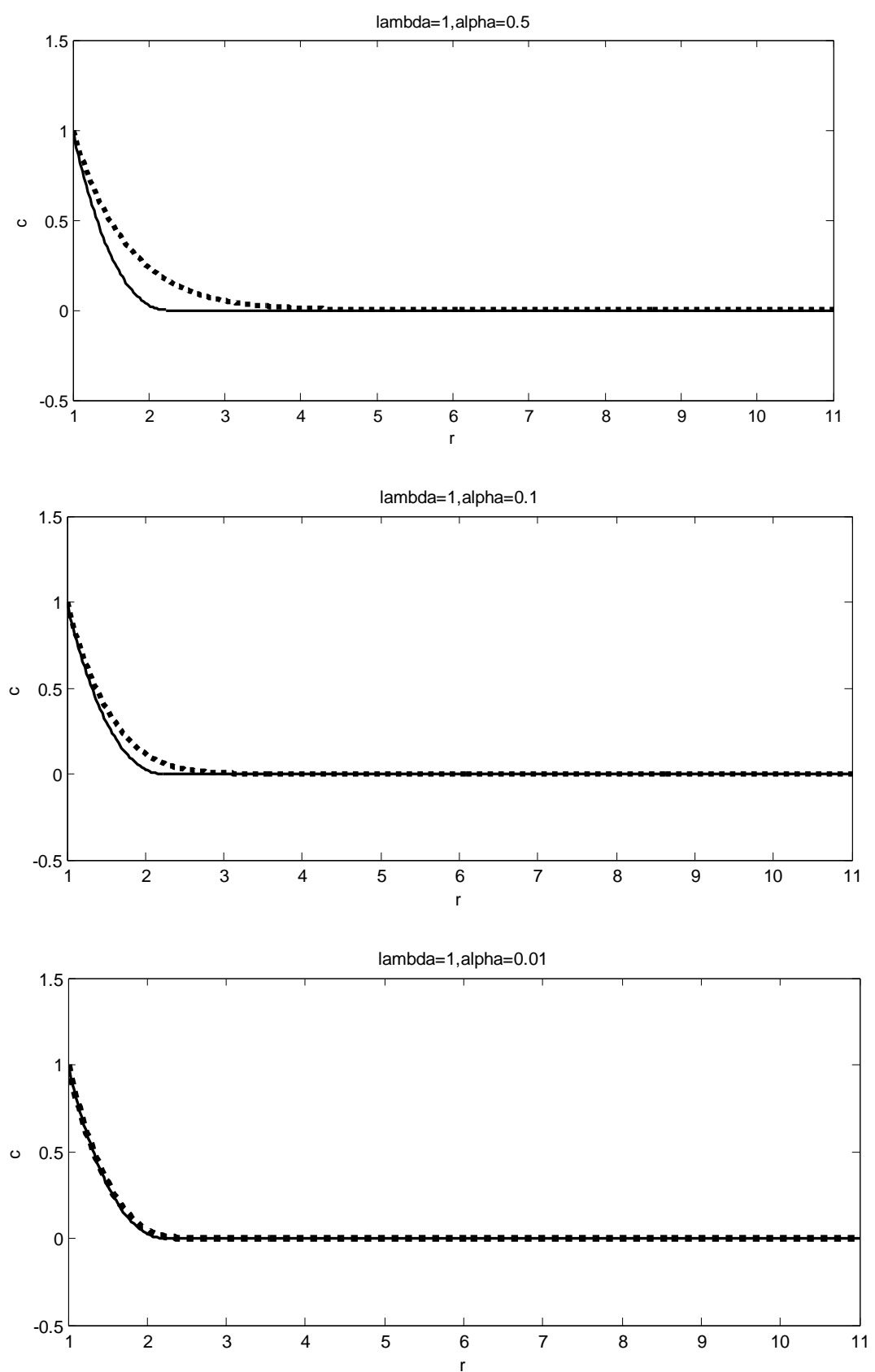

Figure 5 Comparison between numerical solution and leading order approximation for $\lambda=1$ and for various values of $\alpha$. 


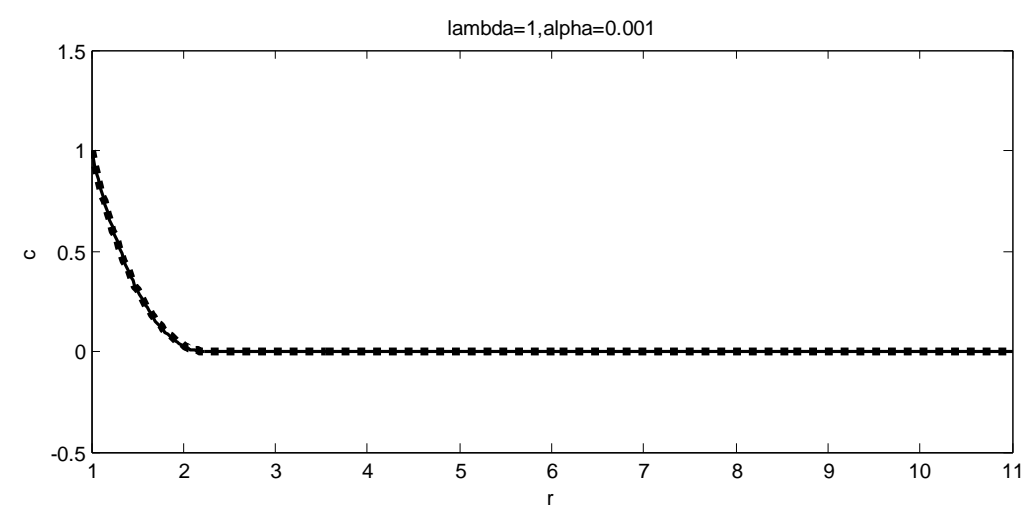

Figure 5 continued. Comparison between numerical solution and leading order approximation for $\lambda=1$ and for various values of $\alpha$.
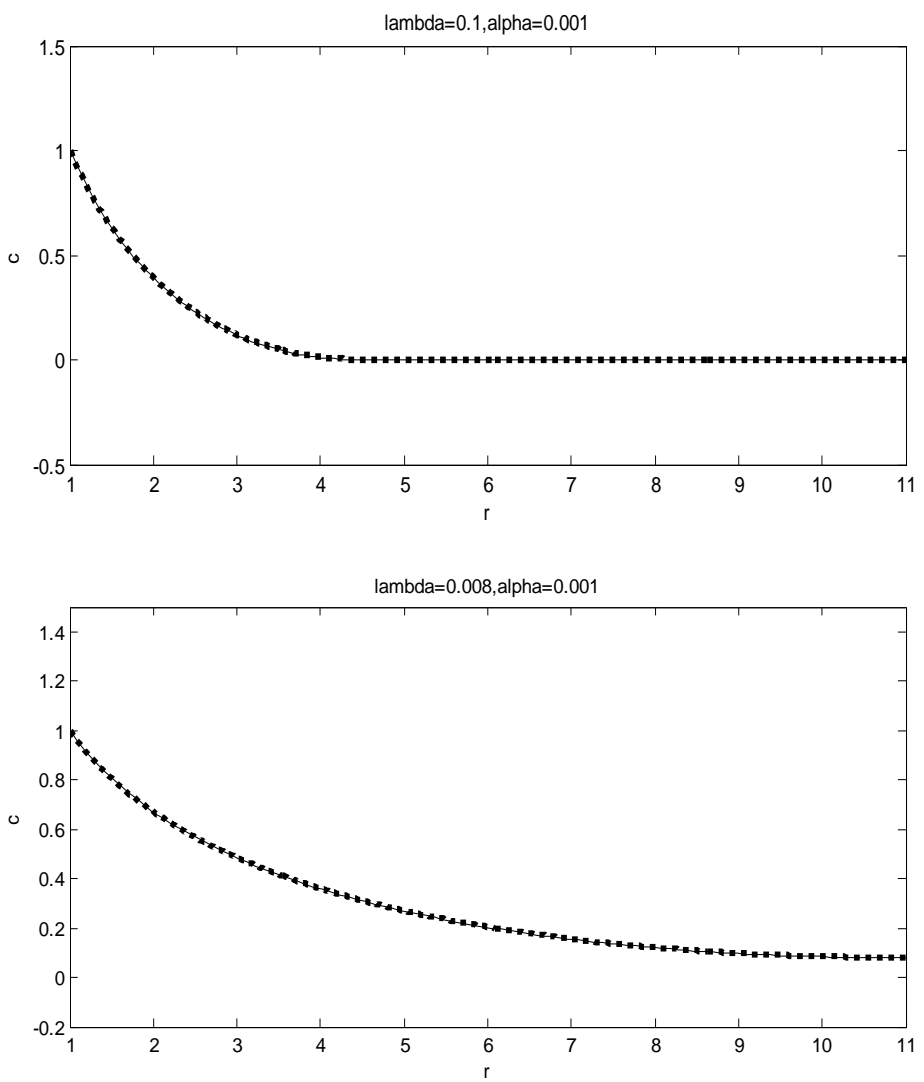

Figure 6 Comparison between numerical solution and leading order approximation for $\alpha=0.001$ and for various values of $\lambda$. 


\section{Conclusions}

We solved the equation for oxygen distribution in the tissue region for the steady state case, analytically and numerically. We studied the case when the Michaelis constant is very small compared to the oxygen concentration in the tissue region. The bound of the value of $\lambda$, representing the ratio of the oxygen reaction rate in the tissue to the concentration of oxygen in the capillary region, was determined. This parameter is important for distributing the oxygen in the tissue region; if the availability of oxygen in the tissue region is much larger than the oxygen reaction rate, then the concentration of oxygen in the tissue will never reach zero; there exists a critical distance above which the region suffers from oxygen deficiency. The other way around, when the availability is smaller than the oxygen rate, the oxygen concentration only asymptotically tends to zero.

\section{Acknowledgements}

The authors would like to thank the reviewers for their careful reading and invaluable suggestions.

\section{Nomenclature}

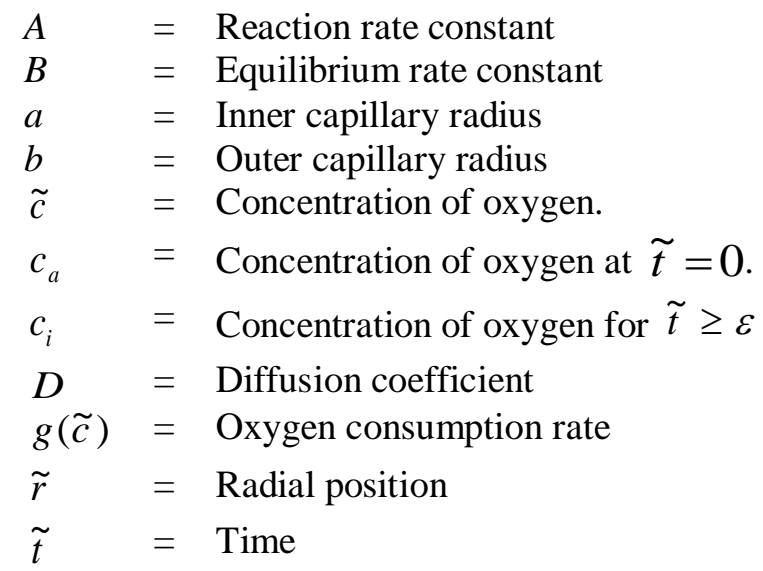

\section{References}

[1] Middleman, S., Transport Phenomena in the Cardiovascular System, John Wiley Sons Inc., pp. 116-140, 1972.

[2] Krogh, A., The Number and Distribution of Capillaries in Muscles with Calculations of The Oxygen Pressure Head Necessary for Supplying Tissue, J. Physiol. (London), 52, pp. 409-415, 1919. 
[3] Salathe, E.P. \& Xu, Y.H, Non-Linear Phenomena in Oxygen Transport To Tissue, J. of Mathematical Biology, 30(2), pp. 151-160, 1991.

[4] Titkombe, M.S. \& Ward, M.J., An Asymptotic Study of Oxygen Transport from Multiple Capillaries to Skeletal Muscle Tissue, SIAM J. Appl. Math., 60(5), pp. 1767-1788,2000.

[5] Salathe, E.P., Mathematical Analysis of Oxygen Concentration in a Two Dimensional Array off Capillaries, J. Math. Biology, 46(4), pp. 287-308, 2003.

[6] Mikelić , M.P., A Diffusion-Consumption Problem for Oxygen in a Living Tissue Perfused by Capillaries, Nonlinear Differ. Equ. Appl. (NoDEA), 13(3), pp. 349-367, 2006.

[7] Devlin, T.M., Textbook of Biochemistry with Clinical Correlations, John Willey \& Sons, pp. 126-129, 1982.

[8] Holmes, M.H., Introduction to Perturbation Method, Springer-Verlag, pp. 47-73, 1959.

[9] Mathews, J.H. \& Fink, K.D., Numerical Methods Using Matlab, PrenticeHall, pp. 505-533, 1999.

[10] Logan, J.D., Applied Partial Differential Equation, Springer-Verlag, pp. 135-138, 1998.

[11] Mattheij, R.M.M., Rienstra, S.W., \& ten ThijeBoonkkamp, J.H.M., Partial Differential Equations: Modeling, Analysis, Computation, 16, SIAM, 2005. 